\title{
Topological interface states mediated by spontaneous symmetry breaking
}

\author{
Roman S. Savelev, ${ }^{1}$ Maxim A. Gorlach, ${ }^{1}$ and Alexander N. Poddubny ${ }^{1,2, *}$ \\ ${ }^{1}$ ITMO University, Saint Petersburg 197101, Russia \\ ${ }^{2}$ Ioffe Institute, Saint Petersburg 194021, Russia
}

(Received 10 February 2018; published 16 July 2018)

\begin{abstract}
We propose a one-dimensional nonlinear system of coupled anharmonic oscillators that dynamically undergoes a topological transition switching from the disordered and topologically trivial phase to the nontrivial one due to the spontaneous symmetry breaking. The topological transition is accompanied by the formation of the topological interface state in the spectrum of linearized excitations of the equilibrium phase. Our findings thus highlight the potential of the nonlinear systems for hosting the topological phases and uncover a fundamental link between the spontaneous-symmetry-breaking mechanism and topological interface states.
\end{abstract}

DOI: 10.1103/PhysRevB.98.045415

\section{INTRODUCTION}

Spontaneous symmetry breaking in nonlinear systems is one of the pivotal concepts of modern science and has important implications for high-energy physics [1,2], the physics of condensed matter [3], nonlinear optical systems [4], Bose-Einstein condensates [5,6], and metamaterials [7]. Spontaneous symmetry breaking in a condensed-matter system is often accompanied by a second-order phase transition, e.g., from a paramagnetic to a ferromagnetic state [8].

An interesting question is whether it is possible to realize a topological phase transition from a trivial to a gapped nontrivial phase. One could expect that the resulting spontaneously broken phase would host topologically nontrivial linear excitations [9]. Topological edge or interface states of electrons [9], light [10-13], and sound [14] have recently received much attention due to their prospects for realization of disorder-robust oneway transport of information. Presently, the interest is shifting towards topological states in nonlinear and interacting systems promising higher tunability and richer fundamental physics [15-23]. However, there is still no clear recipe to realize a nonlinear system with edge or interface states between topologically distinct domains appearing due to spontaneous symmetry breaking. Harnessing the spontaneous nature of the transition would ensure dynamical and low-energy-cost reshaping of topologically trivial potential landscapes into the nontrivial ones and vice versa.

In this paper, we examine the spontaneous formation of interface excitations in the linearized spectrum of the periodic array of nonlinear mechanical oscillators with anharmonic repulsive coupling. We show that after the Peierls-like symmetrybreaking transition [24] an initially disordered system can form topologically distinct regions with linear topological states localized at the domain walls. In other words, the repulsioninduced symmetry breaking gaps the spectrum and generates the topological interface states from the disorder.

*poddubny@coherent.ioffe.ru
The proposed mechanism is qualitatively different from the formation of topological solitons [25], i.e., boundaries between topologically distinct phases, studied across different domains ranging from early-universe physics [26] to liquid helium [27], liquid crystals [28], Bose-Einstein condensates [18,19,29], and mechanical systems [30]. Namely, we aim for topological edge states in the band gap centered at nonzero frequency. These are in stark contrast to both static topological solitons and zero-frequency localized modes of the linearized spectrum of topological solitons [25]. For example, mechanical soliton modes in Ref. [30] appear at zero frequency and acquire nonzero kinetic energy only due to anharmonic effects. In our case the localized solutions have inherently nonzero frequency even in the linearized regime.

The considered spontaneous nonlinear interface state formation in an initially symmetric system is also distinct from one occurring in an intrinsically asymmetric nonlinear system $[15,16]$. It is also different from the case where the potential topology is formed due to the external pump $[17,31,32]$ or magnetic field [33].

The proposed concept is quite general and has implications beyond nonlinear mechanical oscillator arrays $[34,35]$, providing insights into the physics of zigzag and helical cold-ion arrays [36,37], buckled mechanical [38-40] and optomechanical [41] structures, bifurcations in superconducting circuits [42], nanowires $[43,44]$, and nonlinear quantum optics $[22,23]$.

\section{EQUILIBRIUM CONFIGURATIONS AND THE SPECTRUM OF SMALL OSCILLATIONS}

The considered system (Fig. 1) is based on the array of identical anharmonic oscillators [Fig. 1(c)] with double-well on-site potential [Fig. 1(a)] and anharmonic coupling between the nearest neighbors [Fig. 1(b)]. The entire array is described by the potential function

$$
\begin{aligned}
U= & \sum_{n=1}^{N}\left(a_{2} y_{n}^{2}+a_{4} y_{n}^{4}\right)+\sum_{n=1}^{N-1}\left[b_{2}\left(y_{n}-y_{n+1}\right)^{2}\right. \\
& \left.+b_{4}\left(y_{n}-y_{n+1}\right)^{4}\right],
\end{aligned}
$$




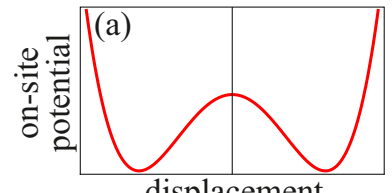

displacement

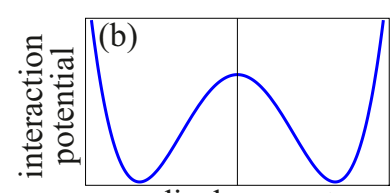

displacement
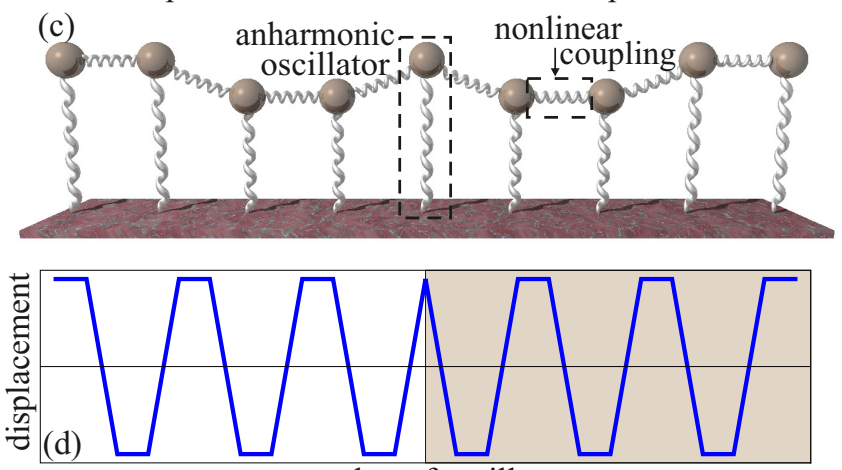

number of oscillator

FIG. 1. An array of anharmonic oscillators with nonlinear coupling between them. (a) On-site potential for the individual oscillator. (b) Interaction potential for two neighboring oscillators. (c) Schematic of the system under study. (d) Equilibrium state of the oscillators after sufficiently long evolution time (tetramer static state) with a domain wall present.

where $y_{n}(t)$ is $n$th oscillator displacement; $a_{2}, a_{4}$ and $b_{2}, b_{4}$ are on-site and intersite force constants, respectively. The terms $\propto a_{4}$ and $\propto b_{4}$ describe the anharmonicity of the potential.

As detailed in Appendix A, one of the stable equilibrium states of such a system is the tetramer static state with a period of 4 when equilibrium displacements of oscillators $y_{n}^{(0)}$ satisfy the conditions

$$
y_{4 n+1}^{(0)}=y_{4 n+2}^{(0)}=-y_{4 n+3}^{(0)}=-y_{4 n+4}^{(0)}=v_{0},
$$

as schematically sketched in Fig. 1(d). The linearized spectrum of small oscillations in the vicinity of this equilibrium state reproduces the Su-Schrieffer-Heeger model (SSH) describing tunneling-coupled arrays with alternating strong and weak tunneling links $[9,42,45-50]$. Hence, the linear spectrum contains interface states localized at the domain wall.

Besides tetramer static states, the system supports also monomer and dimer static configurations with $y_{n}^{(0)}=v_{M}$ and $y_{2 n}^{(0)}=-y_{2 n+1}^{(0)}=v_{D}$, respectively. However, as discussed in Appendixes A and B, small oscillations in the vicinity of these equilibrium states do not reproduce the physics of the SSH model and do not yield any topological states.

Consequently, the tetramer structure is the simplest mechanical realization of the spontaneously induced topological interface states. The equilibrium displacements $v_{0}$ in the tetramer state are found from the condition $\partial U / \partial y_{n}=0$ :

$$
v_{0}= \pm \sqrt{-\frac{a_{2}+2 b_{2}}{2 a_{4}+16 b_{4}}} .
$$

The stability condition imposes an additional inequality on the second derivatives of the potential function (see Appendix C). Furthermore, to ensure that the tetramer static state still persists even in the case of a finite array, one more additional condition

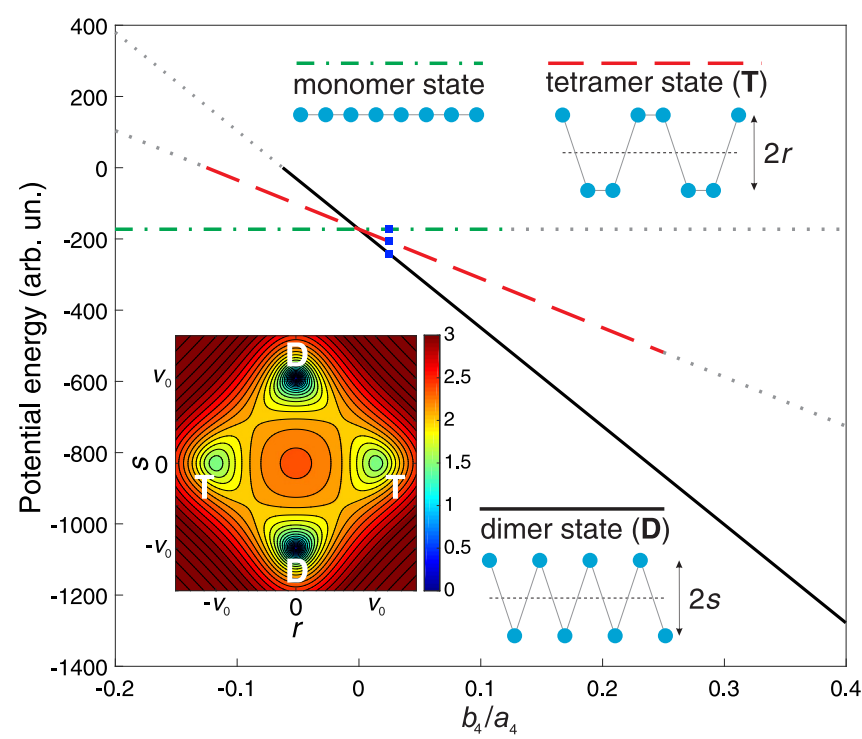

FIG. 2. Potential energy of equilibrium states for the array of anharmonic oscillators as a function of the ratio $b_{4} / a_{4}$ with fixed parameters $a_{2}=-12, a_{4}=5 / 6 ; b_{2}$ is determined from the condition Eq. (4). Solid black, dashed red, and dash-dotted green lines correspond to dimer, tetramer, and monomer static states, respectively. Dotted gray lines indicate the values of $b_{4} / a_{4}$, where the corresponding static states are unstable. Blue squares mark the parameters used in the following calculations. Inset: potential energy as a function of relative displacements $r$ and $s$ defined in the text.

should be fulfilled (Appendix B):

$$
b_{2} / a_{2}=4 b_{4} / a_{4} .
$$

Note that with the latter condition the equilibrium displacement $v_{0}$ both for tetramer and dimer static state is the same as for the single anharmonic oscillator: $v_{0}= \pm \sqrt{-a_{2} /\left(2 a_{4}\right)}$.

Tuning the anharmonicity of on-site and coupling potentials given by the coefficients $a_{4}$ and $b_{4}$ enables one to change the ratio between the energies of monomer, dimer, and tetramer states, as indicated in Fig. 2, thus defining the global energy minimum. As a representative example, we choose $a_{2}=-12$, $b_{2}=-6 / 5, a_{4}=5 / 6$ and define $b_{4}=1 / 48$ according to Eq. (4). The energies of the monomer, dimer, and tetramer states in such a case are indicated by blue squares in Fig. 2. For these parameters, both on-site and coupling nonlinearities are described by double-well potentials as depicted in Figs. 1(a) and 1(b). To further visualize the complicated potential landscape and the interplay between the equilibrium states, we plot the potential energy for a special class of states given by the equations $y_{4 n+1}=r+s, y_{4 n+2}=r-s, y_{4 n+3}=-r+s$, $y_{4 n+4}=-r-s$ and characterized by only two parameters, $r$ and $s$. The calculated color map of the potential function shown in an inset in Fig. 2 features two pairs of local potential minima: $r=0, s= \pm v_{0}$ and $s=0, r= \pm v_{0}$, which correspond to dimer and tetramer static configurations, respectively.

At this point, we highlight that the symmetry-breaking transition happening in the system is not fully spontaneous: the topologically nontrivial spectrum emerges if the system reaches the tetramer static configuration but not the dimer one. Therefore, it is the choice of the initial conditions that 
guarantees the relaxation of the array to the local potential minimum corresponding to the tetramer equilibrium configuration.

As a next step, we examine small oscillations in the vicinity of the tetramer static state. We substitute $y_{n}=y_{n}^{(0)}+$ $\operatorname{Re}\left(z_{n} e^{-i \omega t}\right)$ in the equations of motion, with $y_{n}^{(0)}$ being the oscillator equilibrium displacement given by Eq. (2) and $z_{n}$ representing the complex amplitude of oscillations near the equilibrium state with frequency $\omega$. Keeping only terms linear in $z_{n}$, we get

$$
\begin{array}{ll}
\left(\omega^{2}-\omega_{0}^{2}+2 i \omega \gamma\right) z_{n}=-J_{1} z_{n+1}-J_{2} z_{n-1} & \text { (odd sites), } \\
\left(\omega^{2}-\omega_{0}^{2}+2 i \omega \gamma\right) z_{n}=-J_{2} z_{n+1}-J_{1} z_{n-1} & \text { (even sites), }
\end{array}
$$

with $J_{1}=2 b_{2}, J_{2}=2 b_{2}+48 b_{4} v_{0}^{2}$ so that for the chosen parameters $J_{2} / J_{1}=-2$. The "eigenfrequency" $\omega_{0}^{2}$ is given by a sum of on-site and intersite contributions:

$$
\omega_{0}^{2}=\omega_{\mathrm{R}}^{2}+J_{1}+J_{2},
$$

with $\omega_{\mathrm{R}}^{2}=2 a_{2}+12 a_{4} v_{0}^{2}$. Thus, in terms of bulk properties, oscillations in the vicinity of the tetramer static state are captured by the SSH model with alternating links $J_{1}$ and $J_{2}$. The equation for the edge oscillator is similar:

$$
\left(\omega^{2}-\omega_{0 e}^{2}+2 i \omega \gamma\right) z_{1}=-J_{1} z_{2}
$$

but the eigenfrequency $\omega_{0 e}$ appears to be modified, $\omega_{0 e}^{2}=$ $\omega_{0}^{2}-J_{2}$, which is a consequence of the fact that the edge oscillator has fewer neighbors. For that reason, even if the array is terminated at the weak link edge, large detuning of the edge oscillator prevents the formation of the edge state (Appendix B), in contrast to the conventional SSH case [45]. On the other hand, the topological interface state at the domain wall between the two arrays with opposite dimerizations is still possible in the geometry of Fig. 1(d) (Appendix B).

In order to probe the emergence of the topological order and topological interface state, we analyze the dynamics of $N=120$ oscillators by directly solving nonlinear equations of motion with a small friction term $\gamma=0.05$ included for convergence. Initial displacements $y_{n}(0)$ and velocities $\dot{y}_{n}(0)$ of oscillators were random with uniform distribution in the ranges $\left(y_{n}^{(0)}-\delta y, y_{n}^{(0)}+\delta y\right)$ and $\left(-\dot{\delta}_{y}, \dot{\delta}_{y}\right)$, respectively. Parameters $\delta y=0.4$ and $\dot{\delta}_{y}=1.5$ characterize the degree of randomness. The displacements $y_{n}^{(0)}$ correspond to the pattern depicted in Fig. 1(d) with $v_{0}=2.68$. At each moment of time $t$ the calculated $y_{n}(t)$ were considered as equilibrium displacements, and the spectrum of small oscillations was evaluated. In this way we recovered the evolution of the spectrum presented in Fig. 3(a). It is seen that the spectrum of the system becomes gapped during the evolution, and the edge states appear. Note that the system supports only two interface states: one inside the gap and the other one above the allowed bands, in contrast to the strong-strong defect in the SSH model. This difference comes about as a result of the detuning of the interface oscillator relative to the bulk ones. We are interested in the midgap state, which corresponds to the topological zero-energy state in the SSH model [45]. The calculated displacement distribution depicted in the inset in Fig. 3(a) confirms that this state is indeed localized at the interface.
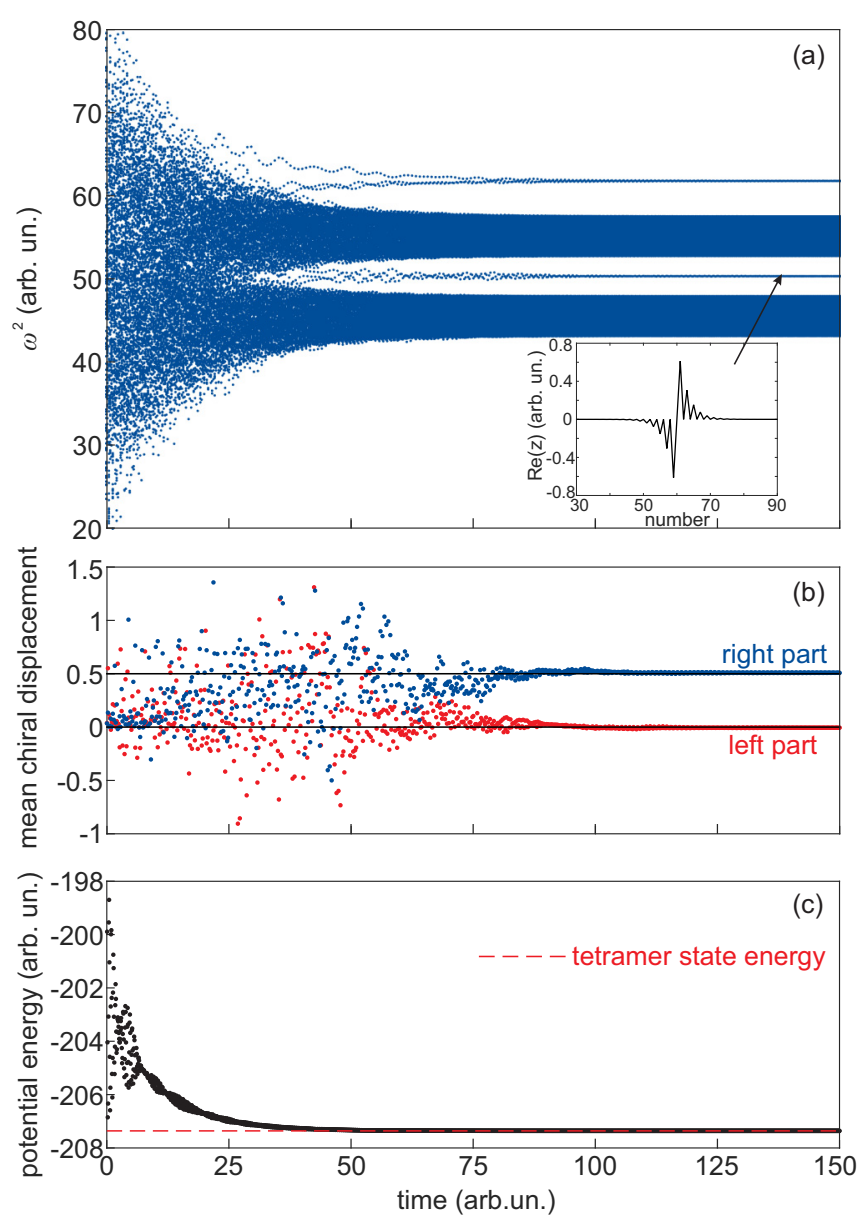

FIG. 3. Time evolution of the array of 120 oscillators. (a) Spectrum of small oscillations. The inset shows the distribution of displacements for the interface mode. (b) Mean chiral displacement calculated for the left (red dots) and right (blue dots) tetramer domains [see Fig. 1(d)]. (c) Potential energy of the system versus evolution time. The red dashed line indicates the potential energy of the tetramer static state.

\section{EMERGENCE OF THE TOPOLOGICAL ORDER: MEAN CHIRAL DISPLACEMENT AND DENSITY-OF-STATES EVOLUTION}

An even more exciting feature is the dynamical emergence of the topological characteristics for an initially disordered system approaching equilibrium. Quite interestingly, the traditional approaches to the topological characterization, for instance, the Zak phase technique [51], can be applied only to the final tetramer configuration of the system, which is periodic. However, these approaches do not allow one to retrace the evolution of the system's topological characteristics in time. To circumvent this difficulty, we have adopted the approach of Refs. [52,53] based on random quantum walks and characterizing the topological properties of the system in terms of mean chiral displacement (MCD).

To evaluate mean chiral displacement, we linearize the equations of motion at each moment of time $t$ and characterize the system by an effective tight-binding Hamiltonian which acts on the wave function $\Psi=\left[\ldots, y_{1}, y_{2}, \ldots\right]^{T}$ and determines the evolution of the system from $t$ to $t+\tau(\tau>0)$. 
Once the wave function of the system $\Psi(t+\tau)$ is found, we evaluate MCD as follows:

$$
\operatorname{MCD}(t, \tau)=\langle\Psi(t+\tau)|\Gamma \mathbf{n}| \Psi(t+\tau)\rangle,
$$

where $\Gamma$ is the matrix of the chiral operator, $\mathbf{n}$ is the matrix of the position operator, so that $\Gamma \mathbf{n}=\operatorname{diag}(\ldots,-1$, $1,0,0,1,-1,2,-2, \ldots)$, and the wave function is localized in an arbitrary unit cell for $\tau=0$. The values of MCD are calculated for the left and right halves of the array independently. For the SSH model, the value of MCD at large times $\tau$ converges to either 0 or 0.5 depending on the choice of the unit cell, which corresponds to the value of the Zak phase being $\gamma=0$ or $\gamma=\pi$, respectively [52]. However, for nonperiodic systems MCD might not converge to any specific value in the limit $\tau \rightarrow \infty$. Such behavior can be observed in Fig. 3(b), where we plotted the values of MCD at $\tau \rightarrow \infty$ as a function of time $t$. At short timescales all oscillators possess relatively large random displacements, and the results for both halves are roughly the same and strongly fluctuate with time; that is, the system does not exhibit any topological properties. In contrast, at larger times $t \gtrsim 100$ the values of $\operatorname{MCD}(t, \tau \rightarrow \infty)$ converge to 0 and 0.5 for the left and right halves of the array, respectively, indicating the formation of topologically distinct phases. This result complements Fig. 3(a), proving the topological origin of the interface state. An interesting additional observation evident from Figs. 3(a) and 3(b) is that the topology measured by the mean chiral displacement "emerges" at the point when the in-gap interface state finally stabilizes in energy, $t \gtrsim 100$ [Fig. 3(c)].

An insightful visualization of the system spectrum in both real and reciprocal space is provided by the density of states (DOS) technique. Real-space- and reciprocal-space-resolved densities of states are calculated as a sum over all eigenstates of the system with a weight that depends on the energy detuning between the energy variable $\omega^{2}$ and the energy of the $m$ th eigenstate $\omega_{0, m}$ :

$$
\begin{aligned}
\operatorname{DOS}_{\text {real }}\left(\omega^{2}, n, t\right) & =\sum_{m=1}^{N} \frac{\left|y_{m}(n, t)\right|}{\sqrt{2 \pi} \sigma} \exp \left(-\frac{\left(\omega_{0, m}^{2}-\omega^{2}\right)^{2}}{2 \sigma^{2}}\right), \\
\operatorname{DOS}_{\text {reciprocal }}\left(\omega^{2}, k, t\right) & =\sum_{m=1}^{N} \frac{\left|\hat{y}_{m}(k, t)\right|}{\sqrt{2 \pi} \sigma} \exp \left(-\frac{\left(\omega_{0, m}^{2}-\omega^{2}\right)^{2}}{2 \sigma^{2}}\right),
\end{aligned}
$$

where $y_{m}$ are eigenvectors of the linearized system at a certain moment $t, \hat{y}_{m}$ is the Fourier transform of $y_{m}$, and $\sigma$ is an auxiliary parameter taken as 0.2 in our calculations.

The calculated real-space- and reciprocal-space-resolved densities of states at the beginning and at the end of evolution are presented in Fig. 4. Full time dynamics can be seen in the movies in the Supplemental Material [54]. At $t=0$ the system is disordered, its eigenmodes are localized [Fig. 4(a)], and the spectrum has no band gap [Fig. 4(b)], in agreement with Fig. 3(a). Examining the density of states in real space at large evolution times [Fig. 4(c)], we observe that the spectrum of the system becomes gapped, and the interface state localized in the middle of the band gap is formed. The density of states in the reciprocal space [Fig. 4(d)] provides clues about the dispersion of the bulk bands, which closely resembles that of the SSH model, further highlighting the topological nature of the studied system.
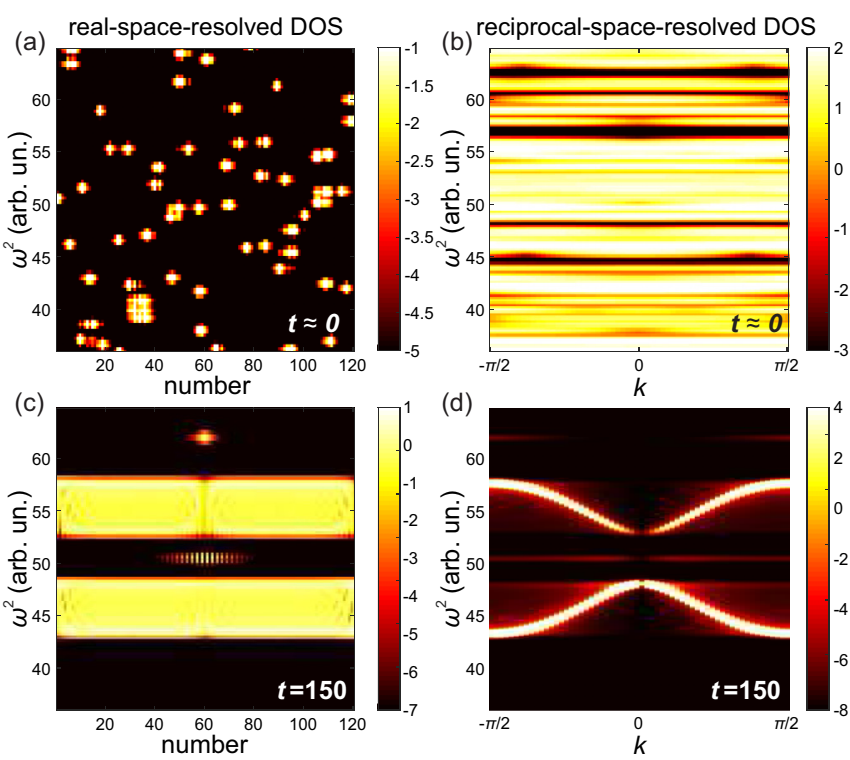

FIG. 4. (a) and (c) Real-space- and (b) and (d) reciprocal-spaceresolved density of states calculated at (a) and (b) $t=0$ and (c) and (d) $t=150$. The final state of the system corresponds to a tetramer static state with domain wall. Here (a)-(c) and (d) are plotted with logarithmic and linear color scales, respectively.

\section{SUMMARY}

To summarize, the fundamental link between spontaneous symmetry breaking and dynamical topological states shown here for a simple mechanical structure applies, in fact, to a wide variety of nonlinear electronic, photonic, and atomic systems. Moreover, the interface states appear to be tolerant of small deviations from Eq. (4). As detailed in Appendix D, possible simple experimental realization of our model is provided by $L C$ circuits with nonlinear capacitive elements in analogy to recent work [55].

\section{ACKNOWLEDGMENTS}

Useful discussions with Yu. S. Kivshar and A. V. Yulin are gratefully acknowledged. Theoretical models were supported by the Russian Science Foundation (Grant No. 16-19-10538). R.S.S. acknowledges partial support by the Russian Foundation for Basic Research (Project No. 16-37-60092 mol a dk). M.A.G. acknowledges partial support by the Foundation for the Advancement of Theoretical Physics "Basis". A.N.P. acknowledges partial support by the Foundation for the Advancement of Theoretical Physics "Basis" and the Russian President Grant No. MD-5791.2018.2.

\section{APPENDIX A: EQUATIONS OF MOTION FOR ANHARMONIC OSCILLATOR ARRAY}

Following the main text, we consider an array of $N$ nonlinearly coupled anharmonic oscillators with a potential function of the form

$$
\begin{aligned}
U= & \sum_{n=1}^{N}\left[a_{2} y_{n}^{2}+a_{4} y_{n}^{4}\right]+\sum_{n=1}^{N-1}\left[b_{2}\left(y_{n}-y_{n+1}\right)^{2}\right. \\
& \left.+b_{4}\left(y_{n}-y_{n+1}\right)^{4}\right]
\end{aligned}
$$


where $y_{n}$ denotes the displacement of the $n$th oscillator and $a_{2}$, $b_{2}, a_{4}$, and $b_{4}$ are the parameters of the model. The dynamics of the system is governed by the equations of motion:

$$
\begin{aligned}
\ddot{y}_{1}+2 \gamma \dot{y}_{1}= & -\partial U / \partial y_{1}=-2 a_{2} y_{1}-4 a_{4} y_{1}^{3} \\
& -2 b_{2}\left(y_{1}-y_{2}\right)-4 b_{4}\left(y_{1}-y_{2}\right)^{3}, \\
\ddot{y}_{n}+2 \gamma \dot{y}_{n}= & -\partial U / \partial y_{n}=-2 a_{2} y_{n}-4 a_{4} y_{n}^{3} \\
& -2 b_{2}\left(2 y_{n}-y_{n-1}-y_{n+1}\right) \\
& -4 b_{4}\left[\left(y_{n}-y_{n-1}\right)^{3}+\left(y_{n}-y_{n+1}\right)^{3}\right], \\
\ddot{y}_{N}+2 \gamma \dot{y}_{N}= & -\partial U / \partial y_{N}=-2 a_{2} y_{N}-4 a_{4} y_{N}^{3} \\
& -2 b_{2}\left(y_{N}-y_{N-1}\right)-4 b_{4}\left(y_{N}-y_{N-1}\right)^{3},
\end{aligned}
$$

where $2 \leqslant n \leqslant N-1$. Equilibrium configurations of the system corresponding to local potential minima are found from Eqs. (A2) by setting to zero the left-hand side. In the case when $a_{2}$ and $a_{4}$ have opposite signs, i.e., the potential function of a single oscillator has a double-well profile, a single anharmonic oscillator has two equilibrium positions, $v_{0}= \pm \sqrt{-a_{2} /\left(2 a_{4}\right)}$. By combining such oscillators into an array and introducing nonlinear coupling between them, it is possible to realize a discrete symmetry-breaking scenario which gives rise to the whole set of static configurations, including a monomer state

$$
y_{n}^{(0)}=v_{M}= \pm \sqrt{-\frac{a_{2}}{2 a_{4}}}
$$

with the potential energy $U_{M}=N \frac{a_{2}}{2} v_{M}^{2}$, a dimer state

$$
y_{2 n}^{(0)}=v_{D}= \pm \sqrt{-\frac{a_{2}+4 b_{2}}{2\left(a_{4}+16 b_{4}\right)}}, \quad y_{2 n+1}^{(0)}=-v_{D}
$$

with the potential energy $U_{D}=N \frac{a_{2}+4 b_{2}}{2} v_{D}^{2}$, and a tetramer state

$$
y_{4 n+1}^{(0)}=y_{4 n+2}^{(0)}=-y_{4 n+3}^{(0)}=-y_{4 n+4}^{(0)}=v_{0},
$$

with

$$
v_{0}= \pm \sqrt{-\frac{a_{2}+2 b_{2}}{2\left(a_{4}+8 b_{4}\right)}}
$$

and potential energy $U_{T}=N \frac{a_{2}+2 b_{2}}{2} v_{0}^{2}$.

The conditions of the existence and stability of these solutions and the corresponding constraints on the parameters of the system are discussed in Appendix C.

Small oscillations. Next, we consider small oscillations in the vicinity of the equilibrium configuration $y_{n}^{(0)}$. For clarity, we omit the $\propto \gamma$ term responsible for dissipation. We represent oscillator displacements as $y_{n}(t)=y_{n}^{(0)}+\operatorname{Re}\left(z_{n} e^{-i \omega t}\right)$, where $\omega$ is the frequency of oscillations. Substituting these expressions into Eqs. (A2) and keeping only terms linear in $z_{n}$, we get

$$
\left(\omega^{2}-\omega_{n 0}^{2}\right) z_{n}+J_{n 1} z_{n+1}+J_{n 2} z_{n-1}=0(2 \leqslant n \leqslant N-1)
$$

and two equations for the edge oscillators:

$$
\begin{aligned}
\left(\omega^{2}-\omega_{10}^{2}\right) z_{1}+J_{11} z_{2} & =0, \\
\left(\omega^{2}-\omega_{N 0}^{2}\right) z_{N}+J_{N 2} z_{N-1} & =0,
\end{aligned}
$$

(a)

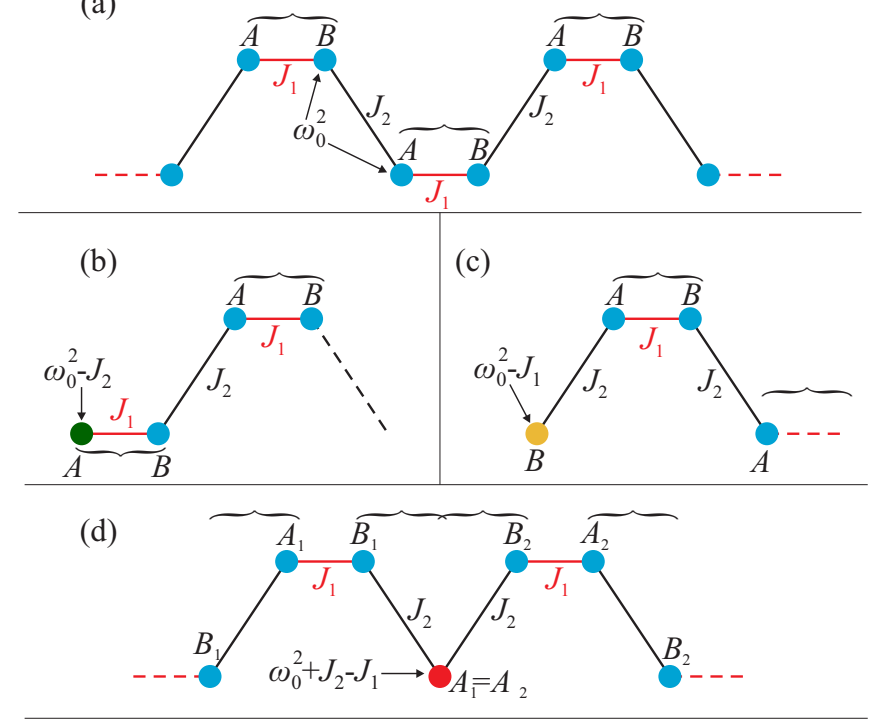

(e)

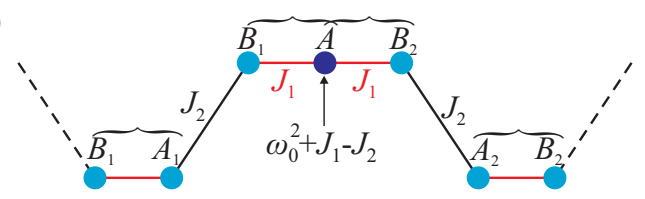

FIG. 5. (a) Scheme of an infinite array in a tetramer equilibrium state. (b) and (c) Scheme of finite arrays in a tetramer equilibrium state with two possible edge terminations. (d) and (e) Scheme of two possible interface configurations between two semi-infinite arrays with different tetramer dimerizations. Brackets indicate the unit cell choice in the left and right domains, $A$ and $B$ denote two sublattices of bipartite array.

where the resonance frequencies of oscillators are represented as a sum of on-site and intersite contributions: $\omega_{n 0}^{2}=\omega_{n R}^{2}+J_{n 1}+J_{n 2}(2 \leqslant n \leqslant N-1), \omega_{10}^{2}=\omega_{1 R}^{2}+J_{11}$, $\omega_{N 0}^{2}=\omega_{N R}^{2}+J_{N 2}$, with $\omega_{n R}^{2}=2 a_{2}+12 a_{4}\left(y_{n}^{(0)}\right)^{2}, \quad J_{n 1}=$ $2 b_{2}+12 b_{4}\left(y_{n+1}^{(0)}-y_{n}^{(0)}\right)^{2}, J_{n 2}=2 b_{2}+12 b_{4}\left(y_{n-1}^{(0)}-y_{n}^{(0)}\right)^{2}$.

Analyzing the linearized equations of motion for an infinite array [i.e., Eq. (A4)], we conclude that monomer and dimer equilibrium configurations are characterized by equal coupling constants $J_{n 1}=J_{n 2}$ due to the fact that $\left(y_{n+1}^{(0)}-y_{n}^{(0)}\right)^{2}=$ $\left(y_{n-1}^{(0)}-y_{n}^{(0)}\right)^{2}$. Therefore, the spectrum of oscillations in the vicinity of monomer and dimer states has a single dispersion band and does not feature any topological states. On the other hand, the coupling constants $J_{n 1}$ and $J_{n 2}$ appear to be different for the tetramer configuration, $\left|J_{n 1}-J_{n 2}\right|=48 b_{4} v_{0}^{2}$, which allows us to map the spectrum of oscillations in the vicinity of the tetramer state onto the Su-Schrieffer-Heeger model.

\section{APPENDIX B: EDGE AND INTERFACE STATES}

Spectrum of small oscillations. In this section, we examine the behavior of small oscillations near the tetramer static configuration in more detail. To obtain a spectrum of such oscillations in an infinite array [Fig. 5(a)], we rewrite Eq. (A4) taking into account the periodicity of the array, $z_{n+2}=z_{n} e^{2 i k}$ :

$$
\begin{gathered}
\left(\omega^{2}-\omega_{0}^{2}\right) A+\left(J_{1}+J_{2} e^{-2 i k}\right) B=0, \\
\left(J_{1}+J_{2} e^{2 i k}\right) A+\left(\omega^{2}-\omega_{0}^{2}\right) B=0,
\end{gathered}
$$




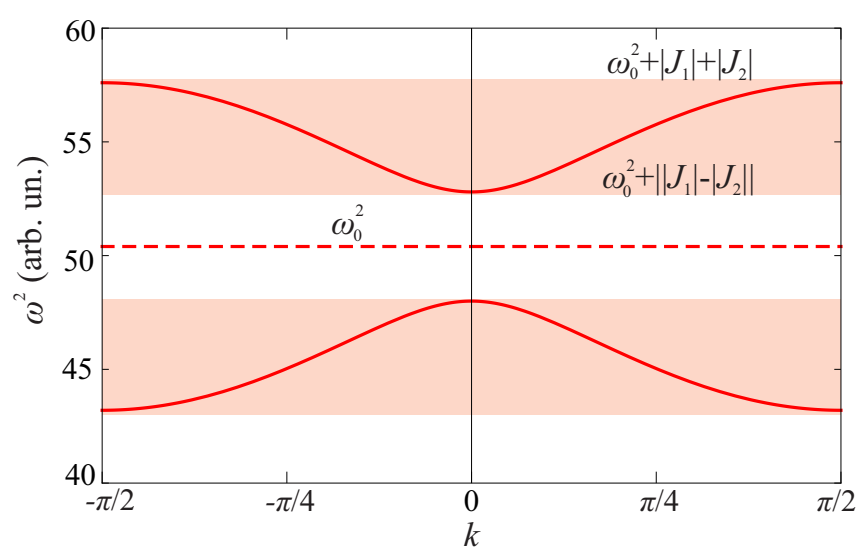

FIG. 6. Spectrum of small oscillations near the tetramer equilibrium state versus wave number $k$ calculated for $a_{2}=-12, b_{2}=$ $-6 / 5, a_{4}=5 / 6, b_{4}=1 / 48$. Shaded regions show spectral positions of the bands. Dashed line marks the center of the bandgap.

where $A$ and $B$ are the amplitudes of the two oscillators in the unit cell, $k$ is the Bloch wave number, and

$$
\begin{gathered}
\omega_{0}^{2}=\omega_{R}^{2}+J_{1}+J_{2}, \\
\omega_{R}^{2}=2 a_{2}+12 a_{4} v_{0}^{2}, \\
J_{1}=2 b_{2}, \\
J_{2}=2 b_{2}+48 b_{4} v_{0}^{2} .
\end{gathered}
$$

Hence, the spectrum of oscillations is given by

$$
\omega^{2}=\omega_{0}^{2} \pm \sqrt{J_{1}^{2}+J_{2}^{2}+2 J_{1} J_{2} \cos (2 k)}
$$

and has a gap. The spectrum calculated for parameter values $a_{2}=-12, b_{2}=-6 / 5, a_{4}=5 / 6, b_{4}=1 / 48$ (as in the main text) is plotted in Fig. 6.

Tetramer configuration in finite arrays. As a next step, we find the conditions which guarantee that tetramer configuration is possible not only for infinite periodic arrays but also for the finite ones. Such conditions ensure that equilibrium displacements of oscillators at the edge are equal to those in the bulk, $\pm v_{0}$. Two possible terminations of the array are shown in Figs. 5(b) and 5(c). In each case we require that the local potential minimum $\partial U / \partial y_{1}=0$ is achieved for $y_{1}= \pm v_{0}$. Making use of Eqs. (A2), we conclude that an extra condition

$$
2 a_{2} v_{0}+4 a_{4} v_{0}^{3}=0
$$

is required for the array termination shown in Fig. 5(b). For the termination in Fig. 5(c) no extra conditions are needed. Equation (B7) can be also presented as

$$
b_{2} / a_{2}=4 b_{4} / a_{4}
$$

which coincides with Eq. (4).

Edge states. If the condition (B8) is fulfilled, the equation for the first oscillator takes the form

$$
\begin{gathered}
\left(\omega^{2}-\omega_{0}^{2}+J_{2}\right) A+J_{1} B=0, \\
\left(\omega^{2}-\omega_{0}^{2}+J_{1}\right) B+J_{2} A e^{2 i k}=0
\end{gathered}
$$

for the terminations of the array in Figs. 5(b) and 5(c), respectively. Combining Eqs. (B9) and (B1) for the termination in Fig. 5(b) and Eqs. (B10) and (B1) for the termination in Fig. 5(c), we prove that the edge states are absent in both cases. Eventually, this happens due to large nonlinear detuning of the edge oscillator.

Interface states. The system, however, can possess states localized at the interface between two different tetramer domains. Two possible types of interface are depicted in Figs. 5(d) and 5(e). To ensure that the equilibrium displacements of interface oscillators are equal to $\pm v_{0}$, we need to enforce the conditions

$$
\begin{aligned}
2 a_{2} v_{0}+4 a_{4} v_{0}^{3}+8 b_{2} v_{0}+64 b_{4} v_{0}^{3} & =0, \\
2 a_{2} v_{0}+4 a_{4} v_{0}^{3} & =0
\end{aligned}
$$

for the interfaces in Figs. 5(d) and 5(e), respectively. Using the expression for $v_{0}$, Eq. (A3), we deduce the same condition, (B8), as derived above for edge oscillator [Fig. 5(b)]. Note that once the condition (B8) is fulfilled, the equilibrium displacements of oscillators, their resonance frequencies, and coupling strengths reduce to

$$
\begin{gathered}
v_{0}=\sqrt{-\frac{a_{2}}{2 a_{4}}}, \\
\omega_{0}^{2}=\omega_{R}^{2}-2 b_{2}, \\
\omega_{R}^{2}=-4 a_{2}, \\
J_{1}=2 b_{2}, \\
J_{2}=-4 b_{2} .
\end{gathered}
$$

Small oscillations of the system in Fig. 5(d) are governed by the equations

$$
\begin{aligned}
\left(\omega^{2}-\omega_{0}^{2}-J_{2}+J_{1}\right) A+J_{2}\left(B_{1}+B_{2}\right) & =0, \\
\left(\omega^{2}-\omega_{0}^{2}\right) A_{1}+B_{1}\left[J_{1} \exp (-2 i k)+J_{2}\right] & =0, \\
\left(\omega^{2}-\omega_{0}^{2}\right) B_{1}+A_{1}\left[J_{1} \exp (2 i k)+J_{2}\right] & =0, \\
\left(\omega^{2}-\omega_{0}^{2}\right) A_{2}+B_{2}\left[J_{1} \exp (-2 i k)+J_{2}\right] & =0, \\
\left(\omega^{2}-\omega_{0}^{2}\right) B_{2}+A_{2}\left[J_{1} \exp (2 i k)+J_{2}\right] & =0,
\end{aligned}
$$

where $A=A_{1}=A_{2}$. All solutions of this system split into symmetric and antisymmetric ones due to mirror symmetry of the array. States localized at the interface are characterized by complex $k$ with a positive imaginary part such that $|\exp (2 i k)|<1$.

Searching for an antisymmetric solution $\left(A=0, B_{1}=\right.$ $\left.-B_{2}\right)$, we immediately obtain $\omega^{2}=\omega_{0}^{2}, \quad \exp (-2 i k)=$ $-J_{2} / J_{1}=2$. This is the state localized at the interface between two tetramer configurations, with energy that always resides exactly in the middle of the gap.

The symmetric solution $\left(A \neq 0, \quad B_{1}=B_{2}\right)$ has energy $\omega^{2}=\omega_{0}^{2}+\frac{\left(J_{2}+J_{1} e^{2 i k}\right)\left(J_{1} e^{-2 i k}-J_{2}\right)}{J_{2}-J_{1}}, \quad$ where $e^{-2 i k}=$ $\frac{3\left(J_{2}-J_{1}\right) \pm \sqrt{9\left(J_{1}-J_{2}\right)^{2}+4 J_{1} J_{2}}}{2 J_{1}}$ and the sign of the square root is chosen such that $\left|e^{2 i k}\right|<1$. In our case with $J_{1}=2 b_{2}$ and $J_{2}=-4 b_{2}$ we take the minus sign corresponding to $A / B<0$ and obtain $\omega^{2}=\omega_{0}^{2}-b_{2}(\sqrt{73}+1)$. 
The second type of interface shown in Fig. 5(e) can be analyzed in an analogous way. The equations for this type of interface are obtained from Eqs. (B17) by replacing $J_{2} \leftrightarrow J_{1}$. In such a configuration, the system does not support antisymmetric interface states, whereas one symmetric interface state appears outside the gap.

Overall, the analysis of this section demonstrates that once the condition (B8) is satisfied, the domain wall in Fig. 5(d) can host an antisymmetric interface state which is a direct counterpart of that in the Su-Schrieffer-Heeger model at a strong-strong defect. However, as we verified numerically, the interface state is tolerant of moderate violations of the condition (B8). Specifically, it persists when the ratio $b_{2} a_{4} /\left(4 a_{2} b_{4}\right)$ varies in the range between 0.8 and 1.3. Thus, the realization of the interface state does not necessarily require fine tuning of the system parameters.

\section{APPENDIX C: CONDITIONS OF THE EXISTENCE AND STABILITY OF THE STATIONARY STATES}

Taking into account the relation (B12), we find out that all considered equilibrium states exist provided the parameters $a_{2}$ and $a_{4}$ have opposite signs; that is, on-site potential has a double-well profile.

The stability of the solutions can be checked by calculating the sign of the minimal value of $\omega^{2}$ in the spectrum of small oscillations. If it is positive, the static configuration is stable, and small perturbations can cause oscillations only near static positions. However, if the minimal value of $\omega^{2}$ becomes negative, an instability in the form of exponentially growing solutions may occur. The boundaries of excitation spectra for all three static configurations are summarized as follows: for the monomer state,

$$
-4\left(a_{2}-b_{2}\right)-4\left|b_{2}\right| \leqslant \omega^{2} \leqslant-4\left(a_{2}-b_{2}\right)+4\left|b_{2}\right| .
$$

For the dimer state,

$$
-4\left(a_{2}+2 b_{2}\right)-8\left|b_{2}\right| \leqslant \omega^{2} \leqslant-4\left(a_{2}+2 b_{2}\right)+8\left|b_{2}\right| \text {. }
$$

For the tetramer state,

$$
\begin{aligned}
& -4\left(a_{2}+b_{2} / 2\right)-6\left|b_{2}\right| \leqslant \omega^{2} \leqslant-4\left(a_{2}+b_{2} / 2\right)-2\left|b_{2}\right|, \\
& -4\left(a_{2}+b_{2} / 2\right)+2\left|b_{2}\right| \leqslant \omega^{2} \leqslant-4\left(a_{2}+b_{2} / 2\right)+6\left|b_{2}\right| .
\end{aligned}
$$

Specifically, Eq. (C3) shows that the positive sign of $a_{2}$ always leads to instability of the tetramer state. Therefore, we consider $a_{2}<0, a_{4}>0$. Furthermore, the ratios $b_{2} / a_{2}$ and $b_{4} / a_{4}$ have the same sign due to the condition (B8). Thus, the stability criteria of equilibrium states read

$$
\begin{aligned}
& b_{4} / a_{4}<1 / 8, \\
& b_{4} / a_{4}>-1 / 16, \\
& -1 / 8<b_{4} / a_{4}<1 / 4
\end{aligned}
$$

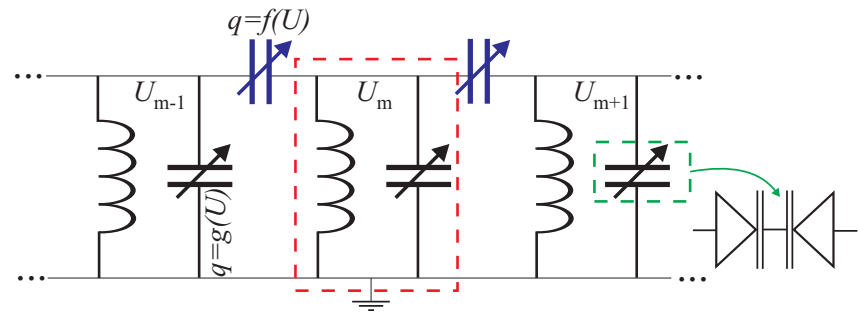

FIG. 7. Possible experimental realization of our proposal: transmission line based on $L C$ circuits with nonlinear capacitive elements (varactors).

for the monomer, dimer, and tetramer states, respectively. The values of the ratio $b_{4} / a_{4}$ that correspond to unstable solutions are shown in Fig. 2 with dotted gray lines for all static configurations.

\section{APPENDIX D: POSSIBLE EXPERIMENTAL REALIZATION WITH LC CIRCUITS}

One of the conceptually simple experimental realizations of the proposed system is provided by $L C$ circuits with inserted nonlinear capacitive elements as depicted in Fig. 7. Nonlinear capacitive elements can be implemented using commercially available varactors with a capacitance that depends on applied voltage. By choosing back-to-back orientation of identical varactors, we ensure that the second-order nonlinearity vanishes, and to the leading order charge stored by such a nonlinear element is related to applied voltage via

$$
\begin{aligned}
& f(U)=\tilde{B}_{1} U+\tilde{B}_{3} U^{3}, \\
& g(U)=\tilde{A}_{1} U+\tilde{A}_{3} U^{3},
\end{aligned}
$$

where functions $f$ and $g$ refer to different nonlinear elements (e.g., based on the different varactor types).

Using Kirchhoff's circuit laws, it is straightforward to verify that the voltages $U_{m}$ in the nodes of the transmission line (Fig. 7) satisfy the following system of equations:

$$
\begin{aligned}
\varepsilon U_{m}= & -A_{3} U_{m}^{3}-B_{1}\left[2 U_{m}-U_{m-1}-U_{m+1}\right] \\
& -B_{3}\left[\left(U_{m}-U_{m-1}\right)^{3}+\left(U_{m}-U_{m+1}\right)^{3}\right],
\end{aligned}
$$

where $\varepsilon=A_{1}-\omega^{-2}, \omega$ is the frequency of monochromatic excitation, $A_{n}=L \tilde{A}_{n}$, and $B_{n}=L \tilde{B}_{n}$, with $n=1,3$. Comparing Eq. (D3) with Eq. (A2), which describes the array of anharmonic oscillators, we observe one-to-one correspondence with slightly different identification of the "energy" variable $\varepsilon$.

To summarize, we believe that our proposal of topological interface states induced by spontaneous symmetry breaking is not only of fundamental interest but can also be readily implemented experimentally.
[1] P. W. Higgs, Broken Symmetries and the Masses of Gauge Bosons, Phys. Rev. Lett. 13, 508 (1964).

[2] S. Chatrchyan et al. (CMS Collaboration), Observation of a new boson at a mass of $125 \mathrm{GeV}$ with the CMS experiment at the LHC, Phys. Lett. B 716, 30 (2012).
[3] C. N. Yang, The Spontaneous Magnetization of a Two-Dimensional Ising Model, Phys. Rev. 85, 808 (1952).

[4] G. Herring, P. G. Kevrekidis, B. A. Malomed, R. CarreteroGonzález, and D. J. Frantzeskakis, Symmetry breaking in 
linearly coupled dynamical lattices, Phys. Rev. E 76, 066606 (2007).

[5] E. A. Ostrovskaya, Y. S. Kivshar, M. Lisak, B. Hall, F. Cattani, and D. Anderson, Coupled-mode theory for Bose-Einstein condensates, Phys. Rev. A 61, 031601 (2000).

[6] I. L. Aleiner, B. L. Altshuler, and Y. G. Rubo, Radiative coupling and weak lasing of exciton-polariton condensates, Phys. Rev. B 85, 121301 (2012).

[7] M. Liu, D. A. Powell, I. V. Shadrivov, M. Lapine, and Y. S. Kivshar, Spontaneous chiral symmetry breaking in metamaterials, Nat. Commun. 5, 4441 (2014).

[8] L. D. Landau and E. M. Lifshitz, Statistical Physics, Part I, Course of Theoretical Physics, Vol. 5 (Pergamon Press, Oxford, 1980).

[9] B. A. Bernevig and T. L. Hughes, Topological Insulators and Topological Superconductors (Princeton University Press, Princeton, NJ, 2013).

[10] L. Lu, J. D. Joannopoulos, and M. Soljačić, Topological photonics, Nat. Photonics 8, 821 (2014).

[11] L. Lu, J. D. Joannopoulos, and M. Soljačić, Topological states in photonic systems, Nat. Phys. 12, 626 (2016).

[12] A. B. Khanikaev and G. Shvets, Two-dimensional topological photonics, Nat. Photonics 11, 763 (2017).

[13] T. Ozawa, H. M. Price, A. Amo, N. Goldman, M. Hafezi, L. Lu, M. Rechtsman, D. Schuster, J. Simon, O. Zilberberg, and I. Carusotto, Topological photonics, arXiv:1802.04173 (2018).

[14] S. D. Huber, Topological mechanics, Nat. Phys. 12, 621 (2016).

[15] Y. Hadad, A. B. Khanikaev, and A. Alù, Self-induced topological transitions and edge states supported by nonlinear staggered potentials, Phys. Rev. B 93, 155112 (2016).

[16] Y. Hadad, V. Vitelli, and A. Alù, Solitons and Propagating Domain Walls in Topological Resonator Arrays, ACS Photonics 4, 1974 (2017).

[17] C.-E. Bardyn, T. Karzig, G. Refael, and T. C. H. Liew, Chiral Bogoliubov excitations in nonlinear bosonic systems, Phys. Rev. B 93, 020502 (2016).

[18] D. D. Solnyshkov, A. V. Nalitov, and G. Malpuech, KibbleZurek Mechanism in Topologically Nontrivial Zigzag Chains of Polariton Micropillars, Phys. Rev. Lett. 116, 046402 (2016).

[19] D. D. Solnyshkov, O. Bleu, B. Teklu, and G. Malpuech, Chirality of Topological Gap Solitons in Bosonic Dimer Chains, Phys. Rev. Lett. 118, 023901 (2017).

[20] D. Leykam and Y. D. Chong, Edge Solitons in NonlinearPhotonic Topological Insulators, Phys. Rev. Lett. 117, 143901 (2016).

[21] X. Zhou, Y. Wang, D. Leykam, and Y. D. Chong, Optical isolation with nonlinear topological photonics, New J. Phys. 19, 095002 (2017).

[22] M. Di Liberto, A. Recati, I. Carusotto, and C. Menotti, Two-body physics in the Su-Schrieffer-Heeger model, Phys. Rev. A 94, 062704 (2016).

[23] M. A. Gorlach and A. N. Poddubny, Topological edge states of bound photon pairs, Phys. Rev. A 95, 053866 (2017).

[24] O.M. Braun and Y.S. Kivshar, The Frenkel-Kontorova Model: Concepts, Methods, and Applications, Physics and Astronomy Online Library (Springer, Berlin, 2004).

[25] N. Manton and P. Sutcliffe, Topological Solitons, Cambridge Monographs on Mathematical Physics (Cambridge University Press, Cambridge, 2007).
[26] T. W. B. Kibble, Topology of cosmic domains and strings, J. Phys. A 9, 1387 (1976).

[27] W. H. Zurek, Cosmological experiments in superfluid helium? Nature (London) 317, 505 (1985).

[28] I. Chuang, R. Durrer, N. Turok, and B. Yurke, Cosmology in the laboratory: Defect dynamics in liquid crystals, Science 251, 1336 (1991).

[29] G. Lamporesi, S. Donadello, S. Serafini, F. Dalfovo, and G. Ferrari, Spontaneous creation of Kibble-Zurek solitons in a Bose-Einstein condensate, Nat. Phys. 9, 656 (2013).

[30] B. G. Chen, N. Upadhyaya, and V. Vitelli, Nonlinear conduction via solitons in a topological mechanical insulator, Proc. Natl. Acad. Sci. U.S.A. 111, 13004 (2014).

[31] V.Peano, C. Brendel, M. Schmidt, and F. Marquardt, Topological Phases of Sound and Light, Phys. Rev. X 5, 031011 (2015).

[32] H. Sigurdsson, G. Li, and T. C. H. Liew, Spontaneous and superfluid chiral edge states in exciton-polariton condensates, Phys. Rev. B 96, 115453 (2017).

[33] A.V. Nalitov, D. D. Solnyshkov, and G. Malpuech, Polariton $\mathbb{Z}$ Topological Insulator, Phys. Rev. Lett. 114, 116401 (2015).

[34] M. Sato, B. E. Hubbard, and A. J. Sievers, Colloquium: Nonlinear energy localization and its manipulation in micromechanical oscillator arrays, Rev. Mod. Phys. 78, 137 (2006).

[35] E. Prodan, K. Dobiszewski, A. Kanwal, J. Palmieri, and C. Prodan, Dynamical Majorana edge modes in a broad class of topological mechanical systems, Nat. Commun. 8, 14587 (2017).

[36] H. Landa, B. Reznik, J. Brox, M. Mielenz, and T. Schaetz, Structure, dynamics and bifurcations of discrete solitons in trapped ion crystals, New J. Phys. 15, 093003 (2013).

[37] R. Nigmatullin, A. del Campo, G. De Chiara, G. Morigi, M. B. Plenio, and A. Retzker, Formation of helical ion chains, Phys. Rev. B 93, 014106 (2016).

[38] N. Stoop, R. Lagrange, D. Terwagne, P. M. Reis, and J. Dunkel, Curvature-induced symmetry breaking determines elastic surface patterns, Nat. Mater. 14, 337 (2015).

[39] J. Paulose, A. S. Meeussen, and V. Vitelli, Selective buckling via states of self-stress in topological metamaterials, Proc. Natl. Acad. Sci. U.S.A. 112, 7639 (2015).

[40] A. Rafsanjani and K. Bertoldi, Buckling-Induced Kirigami, Phys. Rev. Lett. 118, 084301 (2017).

[41] H. Xu, U. Kemiktarak, J. Fan, S. Ragole, J. Lawall, and J. M. Taylor, Observation of optomechanical buckling transitions, Nat. Commun. 8, 14481 (2017).

[42] G. Engelhardt, M. Benito, G. Platero, and T. Brandes, Topologically Enforced Bifurcations in Superconducting Circuits, Phys. Rev. Lett. 118, 197702 (2017).

[43] S. Cheon, T.-H. Kim, S.-H. Lee, and H. W. Yeom, Chiral solitons in a coupled double Peierls chain, Science 350, 182 (2015).

[44] H.-P. Komsa, R. Senga, K. Suenaga, and A. V. Krasheninnikov, Structural distortions and charge density waves in iodine chains encapsulated inside carbon nanotubes, Nano Lett. 17, 3694 (2017).

[45] S.-Q. Shen, Topological Insulators: Dirac Equation in Condensed Matters (Springer, Berlin, 2012).

[46] N. Malkova, I. Hromada, X. Wang, G. Bryant, and Z. Chen, Observation of optical Shockley-like surface states in photonic superlattices, Opt. Lett. 34, 1633 (2009). 
[47] H. Schomerus, Topologically protected midgap states in complex photonic lattices, Opt. Lett. 38, 1912 (2013).

[48] A. P. Slobozhanyuk, A. N. Poddubny, A. E. Miroshnichenko, P. A. Belov, and Y. S. Kivshar, Subwavelength Topological Edge States in Optically Resonant Dielectric Structures, Phys. Rev. Lett. 114, 123901 (2015).

[49] L. Zhang, W. Xie, J. Wang, A. Poddubny, J. Lu, Y. Wang, J. Gu, W. Liu, D. Xu, X. Shen, Y. G. Rubo, B. L. Altshuler, A. V. Kavokin, and Z. Chen, Weak lasing in one-dimensional polariton superlattices, Proc. Natl. Acad. Sci. U.S.A. 112, E1516 (2015).

[50] A. V. Nalitov, T. C. H. Liew, A. V. Kavokin, B. L. Altshuler, and Y. G. Rubo, Spontaneous Polariton Currents in Periodic Lateral Chains, Phys. Rev. Lett. 119, 067406 (2017).

[51] J. Zak, Berry's Phase for Energy Bands in Solids, Phys. Rev. Lett. 62, 2747 (1989).
[52] F. Cardano, A. D’Errico, A. Dauphin, M. Maffei, B. Piccirillo, C. de Lisio, G. De Filippis, V. Cataudella, E. Santamato, L. Marrucci, M. Lewenstein, and P. Massignan, Detection of Zak phases and topological invariants in a chiral quantum walk of twisted photons, Nat. Commun. 8, 15516 (2017).

[53] M. Maffei, A. Dauphin, F. Cardano, M. Lewenstein, and P. Massignan, Topological characterization of chiral models through their long time dynamics, New J. Phys. 20, 013023 (2018).

[54] See Supplemental Material at http://link.aps.org/supplemental/ 10.1103/PhysRevB.98.045415 for the full time dynamics of realspace- and reciprocal-space-resolved densities of states.

[55] Y. Hadad, J. C. Soric, A. B. Khanikaev, and A. Alù, Selfinduced topological protection in nonlinear circuit arrays, Nat. Electronics 1, 178 (2018). 